\title{
Flux Sustainment Condition by Beam Ions in Field-Reversed Configurations
}

\author{
Fusaki P. IIZIMA, Ryuji SEKIGUCHI and Toshiki TAKAHASHI \\ Department of Electronic Engineering, Gunma University, 1-5-1 Tenjin-Cho, Gunma 376-8515, Japan
}

(Received 27 August 2013 / Accepted 28 August 2013)

\begin{abstract}
A flux sustainment condition of field-reversed configurations (FRCs) is given in the present paper for the first time. Here, maintenance of the poloidal flux is considered to be accomplished by tangential neutral beam injection (NBI). The beam current of $700 \mathrm{~A}$ is estimated analytically to be required for a flux balance of an FRC with the resistive anomaly factor of 10 .
\end{abstract}

(C) 2013 The Japan Society of Plasma Science and Nuclear Fusion Research

Keywords: field-reversed configuration, NBI, poloidal flux, resistive decay, flux sustainment condition, GradShafranov equation, toroidal electric field

DOI: $10.1585 /$ pfr.8.1203161

Field-Reversed Configurations (FRCs) have extremely high plasma beta and are sustained by only the poloidal field generated by the toroidal plasma current. Since a typical poloidal flux decay time of FRCs formed by a field reversed theta pinch method is about $100 \mu$ s [1], the plasma is needed to drive the toroidal current. NBI is considered as an adequate current drive method for sustainment of the FRCs, because high energy beam ions contribute to stabilize various MHD instabilities [2], in particular the destructive $n=2$ rotational instability [3, 4]. Investigation of fast ion motion provides effective conditions for NBI to improve an FRC performance. Takahashi and Inomoto calculated separately the power deposition profile in the FIX-FRC plasma at Osaka University [5, 6]. Lifschitz carried out Monte Carlo simulation and found a self-consistent beam-plasma equilibrium state [7] calculated by solving the Grad-Shafranov equation with a beam current term [8]. Here, slight interaction between beam and plasma particles is assumed, and therefore the pressure of the beam particles and the friction force between beam ions and electrons are neglected in the beam-plasma equilibrium model. Since the poloidal flux in FRCs suffers from a rapid decay by anomalous phenomena, NBI should also have a role to suppress the decay by an additional flux generated by the beam current. In order to study the flux balance between a resistive decay and flux supply by beam ions, we need a new theoretical model. In the present paper, we will describe a strict condition to keep the poloidal flux constant and show a spatial profile of the time derivative of beam current density necessary for a steady state condition.

A steady state condition with respect to the poloidal magnetic flux represents a situation where the rate of flux supply by beam ions cancels the flux decay rate of the FRC plasma. There exists no toroidal electric field here, and

author'se-mail: t-tak@el.gunma-u.ac.jp hence no radial drift motion would be induced. Suppose that neutral beam particles are injected in the direction tangential to the plasma current, i.e., in the toroidal direction. Then the beam current in an FRC plasma is gradually augmented during tangential NBI. The toroidal beam ion current density $j_{\mathrm{b} \theta}$ relates with the poloidal flux according to the Ampere's law written in the cylindrical coordinates $r$ and $z$ :

$$
r \frac{\partial}{\partial r}\left(\frac{1}{r} \frac{\partial \psi_{\mathrm{b}}}{\partial r}\right)+\frac{\partial^{2} \psi_{\mathrm{b}}}{\partial z^{2}}=-\mu_{0} r j_{\mathrm{b} \theta}
$$

Here, $\psi_{\mathrm{b}}$ is the poloidal flux generated by beam ions alone and effects of displacement current can be neglected because of the non-relativistic nature. The beam ions are gradually accumulated in the core plasma from the beginning of NBI, and the beam ion current will increase with time. Differentiation of Eq. (1) with respect to time gives

$$
r \frac{\partial}{\partial r}\left(\frac{1}{r} \frac{\partial}{\partial r}\left(\frac{\partial \psi_{\mathrm{b}}}{\partial t}\right)\right)+\frac{\partial^{2}}{\partial z^{2}}\left(\frac{\partial \psi_{\mathrm{b}}}{\partial t}\right)=-\mu_{0} r\left(\frac{\partial j_{\mathrm{b} \theta}}{\partial t}\right) .
$$

When a steady state is accomplished by satisfying the strict condition as the resistive decay rate of the poloidal flux balances building-up rate by NBI, we write

$$
\frac{\partial \psi_{\mathrm{p}}}{\partial t}+\frac{\partial \psi_{\mathrm{b}}}{\partial t}=0
$$

where $\psi_{\mathrm{p}}$ is the poloidal flux formed by the external coil and plasma currents. The flux $\psi_{\mathrm{p}}$ is decaying with time by plasma resistivity. In axisymmetric case, the Faraday's law gives

$$
\frac{\partial \psi_{\mathrm{p}}}{\partial t}=-r E_{\mathrm{p} \theta}=-r \eta j_{\mathrm{p} \theta}
$$

where $E_{\mathrm{p} \theta}, j_{\mathrm{p} \theta}$, and $\eta$ are the toroidal electric field induced by the electron motion, the plasma current density in the toroidal direction, and the resistivity. The toroidal electric field in FRC plasmas is usually attributed to an anomalous resistivity. Hence, if the flux balance is strictly satisfied, the increasing rate of the poloidal flux by NBI is 


$$
\frac{\partial \psi_{\mathrm{b}}}{\partial t}=-\frac{\partial \psi_{\mathrm{p}}}{\partial t}=r E_{\mathrm{p} \theta}
$$

When Eq. (5) is satisfied, there exists no toroidal electric field and the radial compression and/or expansion due to an electric drift motion never occur in FRCs. Substituting Eq. (5) in Eq. (2), we obtain a required condition with respect to the beam current density to sustain an FRC plasma at a steady state as

$$
\frac{\partial j_{\mathrm{b} \theta}}{\partial t}=-\frac{1}{\mu_{0}}\left[\frac{\partial}{\partial r}\left(\frac{1}{r} \frac{\partial}{\partial r}\left(r E_{\mathrm{p} \theta}\right)\right)+\frac{\partial^{2} E_{\mathrm{p} \theta}}{\partial z^{2}}\right] .
$$

Equation (6) suggests efficient NBI geometry and required beam current to sustain the plasma. In the present paper, we will show the spatial distribution of $\partial_{t} j_{\mathrm{b} \theta}$.

An equilibrium state of FRC is calculated by solving the Grad-Shafranov equation. A choice of the pressure function determines the current and toroidal electric field profiles. We set the pressure function in the same manner as [9]. After obtaining a Grad-Shafranov equilibrium, we estimate $E_{\mathrm{p} \theta}$ by giving the resistivity as $\eta=A \eta_{\mathrm{cl}}$, where $\eta_{\mathrm{cl}}$ and $A$ are the classical resistivity and anomaly factor, respectively. In our case the electron temperature is assumed to be uniform inside the separatrix, and hence the resistivity is constant. By comparing with the time evolution of excluded flux radius with the NUCTE-III experiment, we set the anomaly factor $A$ to $10[10]$. Calculated results are shown in Fig. 1. Here, $E_{\mathrm{p} \theta}$ is normalized by $\left|\psi_{\mathrm{w}}\right| /\left(r_{\mathrm{w}} \tau\right)$, where $r_{\mathrm{w}}, \psi_{\mathrm{w}}$, and $\tau$ are the radius of the calculation boundary, the flux function at $r_{\mathrm{w}}$, and the typical ion gyration time defined as $m_{\mathrm{i}} r_{\mathrm{w}}^{2} /\left[q_{\mathrm{i}}\left|\psi_{\mathrm{w}}\right|\right]$. In our case, the plasma and beam ions are both deuterium ions. Also, $r_{\mathrm{w}}=0.17 \mathrm{~m}[11], \psi_{\mathrm{w}}=0.16 \times 10^{-2} \mathrm{~Wb}$ and $\tau=0.38 \times 10^{-6}$ sec for Fig. 1 (a), and $\psi_{\mathrm{w}}=0.48 \times 10^{-2} \mathrm{~Wb}$ and $\tau=0.13 \times 10^{-6} \mathrm{sec}$ for Fig. 1 (b). The beam current rise rate $\partial_{t} j_{\mathrm{b} \theta}$ in Fig. 1 is also dimensionless quantity and normalized by $\left|\psi_{\mathrm{w}}\right| /\left(2 \mu_{0} r_{\mathrm{w}}{ }^{3} \tau\right)$, which is $3.4 \times 10^{11} \mathrm{~A} /\left(\mathrm{m}^{2} \cdot \mathrm{sec}\right)$ for Fig. 1 (a) and $3.1 \times 10^{12} \mathrm{~A} /\left(\mathrm{m}^{2} \cdot \mathrm{sec}\right)$ for Fig. 1 (b). When the pressure function is linear function of the flux, the plasma current is linear with the radial distance from the geometric axis, i.e. the rigid rotor profile, as shown in Fig. 1 (a). When the pressure is cubic function of the flux, $j_{\mathrm{p} \theta}$ and $E_{\mathrm{p} \theta}$ become hollow shape and are peaking at the separatrix, as shown in Fig. 1 (b). To show the profile of $\partial_{t} j_{\mathrm{b} \theta}$ more clearly, its color contour in the $r-z$ plane for the case of Fig. 1 (b) is shown in Fig. 2. It is also found from Fig. 2 that $\partial_{t} j_{\mathrm{b} \theta}$ localizes in a very narrow region neighborhood of the separatrix where the toroidal plasma current concentrates. Suppose that the beam current is constant and the beam current density is augmented linearly with time because of accumulation of beam ions in the core plasma, a required beam current can be estimated by

$$
I_{\mathrm{b}}=\left(\int_{S} \partial_{t} j_{\mathrm{b} \theta} \mathrm{d} S\right) \Delta t
$$

where $S$ is the poloidal cross section and $\Delta t$ is the duration of beam current rise to sustain the equilibrium state. When $\Delta t$ is $1 \mu \mathrm{sec}$, a required beam current is $700 \mathrm{~A}$, which is
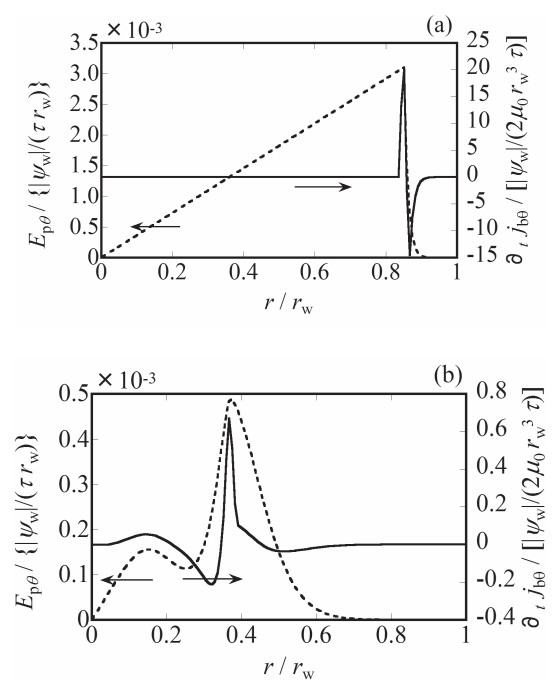

Fig. 1 Radial midplane profile of toroidal electric field (the dotted line) and the current rise rate (the solid line) for (a) the rigid rotor and (b) hollow current profile.

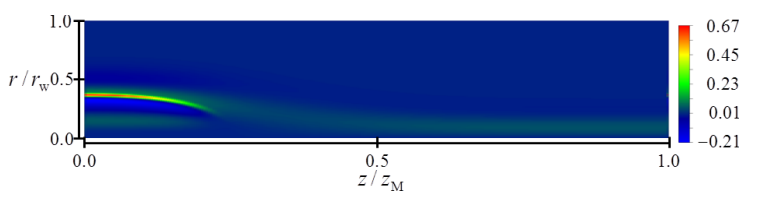

Fig. 2 The color contour of two dimensional profile of the current rise rate.

much higher than usual NBI experiments for FRC plasmas $[12,13]$. If the anomaly factor $A$ reduces to unity, then the needed beam current would be $70 \mathrm{~A}$; it is feasible in the current circumstances.

The obtained $\partial_{t} j_{\mathrm{b} \theta}$ here only shows the momentary profile at an equilibrium state. The equilibrium profile gradually changes as beam ions are accumulated during NBI. Resultantly, $\partial_{t} j_{\mathrm{b} \theta}$ in Eq. (6) changes with time as the current density and associated toroidal electric field profiles changes. Its time evolution will be calculated by a Monte Carlo simulation, and it is targeted as a topic for a future study.

[1] A.L. Hoffman and J.T. Slough, Nucl. Fusion 33, 27 (1993).

[2] T. Asai et al., Phys. Plasmas 10, 3608 (2003).

[3] T. Ii et al., Nucl. Fusion 53, 073002 (2013).

[4] E.V. Belova et al., Phys. Plasmas 13, 056115 (2006).

[5] T. Takahashi et al., Phys. Plasmas 11, 3801 (2004).

[6] M. Inomoto et al., Nucl. Fusion 48, 035013 (2008).

[7] A.F. Lifschitz, R. Farengo and N.R. Arista, Nucl. Fusion 42, 863 (2002).

[8] J.M. Finn and R.N. Sudan, Nucl. Fusion 22, 1443 (1982).

[9] T. Takahashi et al., Phys. Plasmas 11, 3131 (2004).

[10] T. Takahashi et al., Plasma Fusion Res. 2, 008 (2007).

[11] T. Asai et al., Phys. Plasmas 13, 071508 (2006).

[12] M. Tuszewski et al., Phys. Plasmas 19, 056108 (2012).

[13] T. Ii et al., Rev. Sci. Instrum. 83, 083504 (2012). 